\title{
Low Self-Efficacy and High Kinesiophobia Are Associated With Worse Function in Patients With Femoroacetabular Impingement Syndrome
}

\author{
Kate N. Jochimsen, Carl G. Mattacola, Brian Noehren, Kelsey J. Picha, \\ Stephen T. Duncan, and Cale A. Jacobs
}

\begin{abstract}
Context: Femoroacetabular impingement syndrome (FAIS) is a painfully debilitating hip condition disproportionately affecting active individuals. Mental health disorders are an important determinant of treatment outcomes for individuals with FAIS. Selfefficacy, kinesiophobia, and pain catastrophizing are psychosocial factors that have been linked to inferior outcomes for a variety of orthopedic conditions. However, these psychosocial factors and their relationships with mental health disorders, pain, and function have not been examined in individuals with FAIS. Objective: (1) To examine relationships between self-efficacy, kinesiophobia, pain catastrophizing, pain, and function in patients with FAIS and (2) to determine if these variables differ between patients with and without a self-reported depression and/or anxiety. Design: Cross-sectional. Setting: University health center. Participants: Fifty-one individuals with FAIS (42 females/9 males; age 35.7 [11.6] y; body mass index 27.1 [4.9] kg/m²). Main Outcome Measures: Participants completed the Pain Self-Efficacy Questionnaire, Tampa Scale for Kinesiophobia, Pain Catastrophizing Scale, visual analog scale for hip pain at rest and during activity, and the 12-item International Hip Outcome Tool. Self-reported depression and/or anxiety were recorded. The relationships between psychosocial factors, pain, and function were examined using Spearman rank-order correlations. Independent $t$ tests and Mann-Whitney $U$ tests were used to evaluate the effect of self-reported depression and/or anxiety on psychosocial factors, pain and function. Results: The 12-item International Hip Outcome Tool was correlated with pain during activity $(\rho=-.57, P \leq .001)$, Tampa Scale for Kinesiophobia $(\rho=-.52$, $P \leq .001)$, and Pain Self-Efficacy Questionnaire $(\rho=.71, P \leq .001)$. The Pain Self-Efficacy Questionnaire was also correlated with pain at rest $(\rho=-.43, P=.002)$ and pain during activity $(\rho=-.46, P=.001)$. Individuals with self-reported depression and/ or anxiety $(18 / 51 ; 35.3 \%)$ had worse self-efficacy and pain catastrophizing $(P \leq .01)$. Conclusion: Self-reported depression and/ or anxiety, low self-efficacy, and high kinesiophobia were associated with more hip pain and worse function for patients with FAIS. These findings warrant further examination including psychosocial treatment strategies to improve the likelihood of a successful clinical outcome for this at-risk population.
\end{abstract}

Keywords: pain catastrophizing, psychosocial factors, hip pain, depression, anxiety

Femoroacetabular impingement syndrome (FAIS) primarily affects young active individuals and is comprised of limited motion and painful daily activities in the presence of variant hip morphology. ${ }^{1-3}$ Individuals with FAIS often experience hip pain and dysfunction for years prior to receiving the correct diagnosis. ${ }^{4-6}$ Though many patients report improved pain and function following intervention; currently, both surgical and conservative treatments for FAIS are falling short of providing sustained benefit. ${ }^{7-9}$ Conservative treatment including therapy and intraarticular hip injections can improve pain and function in the short term. ${ }^{10}$ However, the vast majority of patients have relapsing pain and

Jochimsen was with the Department of Rehabilitation Sciences, University of Kentucky, Lexington, KY, USA; and is now with the Division of Athletic Training, West Virginia University, Morgantown, WV, USA. Mattacola is with the School of Health and Human Sciences, The University of North Carolina Greensboro, Greensboro, NC, USA. Noehren is with the Division of Physical Therapy, University of Kentucky, Lexington, KY, USA. Picha is with Clinical Anatomy, Department of Health Sciences, A.T. Still University, Mesa, AZ, USA. Duncan is with Adult Reconstruction, Hip Resurfacing, and Hip Preservation, Department of Orthopedic Surgery \& Sports Medicine, University of Kentucky, Lexington, KY, USA. Jacobs is with Orthopedic Research, Department of Orthopedic Surgery \& Sports Medicine, University of Kentucky, Lexington, KY, USA. Jochimsen (kate.jochimsen@hsc. wvu.edu) is corresponding author. functional impairments and thus progress to surgical intervention. ${ }^{11,12}$ At an average of 2 years following hip arthroscopy, 1 in 4 individuals report persistent hip pain and 1 in 3 report unacceptable sports function. ${ }^{13,14}$ One potential barrier to decreasing pain and improving functional outcomes for individuals with FAIS is poor mental health.

Mental health is an important determinant of pain and functional outcomes for individuals with FAIS. ${ }^{13-18}$ Recent cohort studies of 500 and 688 patients, respectively, found self-reported depression and/or anxiety to be a predictor for not achieving acceptable sports function and for persistent postoperative pain 2-year posthip arthroscopy. ${ }^{13,14}$ Additional psychosocial factors such as pain catastrophizing, self-efficacy, and kinesiophobia are key contributors to pain and functional outcomes in orthopedic conditions of the shoulder and knee. ${ }^{19-21}$ However, very limited work has explored their relationships with self-reported depression and anxiety, pain, and functional outcomes for individuals with FAIS.

Pain catastrophizing is a psychosocial factor that involves ruminating on painful experiences, feeling helpless in overcoming painful situations, and magnifying the circumstances surrounding pain or injury. ${ }^{22}$ Pain catastrophizing has been identified as an important mediator between pain and function. ${ }^{23,24} \mathrm{In}$ addition, it is related to worse orthopedic outcomes such as increased disability and persistent pain. ${ }^{25,26}$ Pain self-efficacy is a task- and situation- 
specific psychosocial factor that gauges an individual's confidence in completing tasks despite their current pain. ${ }^{27}$ Self-efficacy is an important determinant of long-term success following total hip arthroplasty and is a mediator in the relationship between pain and disability. ${ }^{28,29}$ Kinesiophobia, or a fear of movement, is associated with not returning to preinjury levels of activity in individuals postanterior cruciate ligament reconstruction. ${ }^{30}$ Moreover, fear has been identified as one of the most common reasons individuals do not return to sport following hip arthroscopy for FAIS. ${ }^{31,32}$

To date, self-efficacy has not been described in patients with FAIS; however, pain catastrophizing and kinesiophobia have been briefly characterized. ${ }^{11,33}$ The first study to examine pain catastrophizing in individuals with FAIS reported a mean Pain Catastrophizing Scale (PCS) score of 23.7 (11.8) in patients with FAIS. ${ }^{11}$ PCS scores range from 0 to 52 with higher scores representing higher levels of pain catastrophizing. ${ }^{22}$ This mean score of 23 is above that of healthy individuals (14.5 [9.3]) and the cutoff for clinically relevant pain catastrophizing $(\geq 19) .{ }^{22,34}$ Unfortunately, the authors did not examine the relationships between pain catastrophizing, pain, and hip-specific outcome scores. The second study reported a mean PCS score of 17.81 (10.13) and a mean Tampa Scale for Kinesiophobia-11 of 26.22 (5.99). ${ }^{33}$ Tampa Scale for Kinesiophobia-11 scores range from 11 to 44 with higher scores representing higher levels of fear. ${ }^{35}$ Patients who failed to achieve a minimally clinically important difference on a hip-specific outcome score 1 year postoperatively had higher pain catastrophizing, but not kinesiophobia, preoperatively. ${ }^{33}$ Collectively, findings from these 2 studies suggest pain catastrophizing may be an important psychosocial factor to consider when treating individuals with FAIS; however, more work needs to be done before clinical recommendations can be made.

The consequences of pain catastrophizing behaviors, deficits in self-efficacy, and increased kinesiophobia on health outcomes in patients with musculoskeletal injuries can be described by the fearavoidance model by Woby et $\mathrm{al}^{36}$ This model postulates that patients respond to pain in 1 of 2 ways: avoidance or confrontation. Psychosocial factors influence whether patients will display avoidance behaviors or confront their pain and rehabilitation. For example, high levels of pain catastrophizing and kinesiophobia may lead the patient down an avoidant path resulting in greater disuse and worse function. Contrarily, low levels of pain catastrophizing and kinesiophobia may lead the patient down a confrontational path resulting in greater engagement in rehabilitation and better function. Self-efficacy has been found to serve as a mediator between kinesiophobia and both pain and functional disability. ${ }^{36}$ Exploring the relationships between pain catastrophizing, self-efficacy, kinesiophobia, pain, and function in individuals with FAIS may shed light on potentially modifiable barriers to optimal recovery.

Current estimates on the prevalence of mental health disorders in individuals with FAIS suggest greater than 1 in 3 patients have comorbid depression and/or anxiety. ${ }^{15,37}$ A 2019 systematic review and meta-analysis of 12 studies concluded that individuals with mental health disorders have significantly worse pain and functional outcomes following hip arthroscopy. ${ }^{18}$ Depression has been associated with higher levels of pain catastrophizing in individuals with chronic pain, ${ }^{38}$ and greater anxiety and depression symptoms are related with worse self-efficacy in individuals with knee osteoarthritis. ${ }^{39}$ However, in individuals with FAIS, psychosocial factors have not been compared between patients with and without self-reported depression and/or anxiety. It is critically important for rehabilitation specialists to examine these differences in order to understand factors influencing treatment responsiveness. In addition, it is an important step in developing effective targeted rehabilitation interventions that motivate interdisciplinary relationships to optimize patient outcomes.

Unlike depression and anxiety which require specialized care from a mental health professional, low self-efficacy, kinesiophobia, and pain catastrophizing are amendable with allied health interventions including pain neuroscience education and goal setting. Currently, these evidence-based interventions are precluded by our gap in knowledge regarding the individual or combined roles psychosocial factors play on clinical outcomes in individuals with FAIS. Therefore, the purposes of this study were to (1) examine the relationships between pain catastrophizing, self-efficacy, and kinesiophobia, pain, and function in patients with FAIS and (2) determine if these variables differ between patients with and without selfreported depression and/or anxiety. We hypothesized that higher pain catastrophizing, lower self-efficacy, and higher kinesiophobia would be related with more severe pain and lower function. In addition, we hypothesized that patients with self-reported depression and/or anxiety would have worse psychosocial health, pain, and function. Because of the high prevalence of comorbid mental health disorders and maladaptive pain coping strategies seen in patients with FAIS patients, identifying the predominant factors associated with pain and functional deficits is necessary in order to develop multidisciplinary strategies to improve the likelihood of successful clinical outcomes.

\section{Methods}

\section{Study Design and Participants}

This project had appropriate approval from the University of Kentucky Institutional Review Board. This cross-sectional study enrolled 82 consecutive patients who provided both oral and written consent. Patients were included if they were diagnosed with FAIS; had closed epiphyseal plates; read and spoke fluent English; and had attempted some type of conservative treatment such as intraarticular cortisone injection, physical therapy, and lifestyle modification. Patients were recruited in person from a single fellowship trained hip preservation surgeon from March 2017 to November 2018. Though treatment decision is not an outcome of interest in this study, it is important to note that some patients went on to surgical intervention, while others continued varied courses of conservative care. Patients who previously underwent hip arthroscopy $(n=5)$ were excluded to create a homogenous population. In addition, as pain was a primary variable of interest, patients with fibromyalgia or complex regional pain syndrome were excluded $(n=1)$. In total, 51 participants had complete data and met all inclusion criteria (Figure 1).

\section{Variables of Interest}

Demographic data and duration of symptoms (months) were recorded. Next, participants completed the PCS, Pain Self-Efficacy Questionnaire (PSEQ), Tampa Scale for Kinesiophobia (TSK), a 10-point visual analog scale (VAS) for hip pain both during rest and activity, and the 12-item International Hip Outcome Tool (iHOT-12). Participants also answered a yes/no question regarding their self-reported mental health (ie, "Do you suffer from anxiety or depression?")

Pain catastrophizing was measured via the 13-item PCS. Participants rated each of the 13 statements on a scale from 0 (not at all) to 4 (all the time). The total PCS score ranges from 0 to 


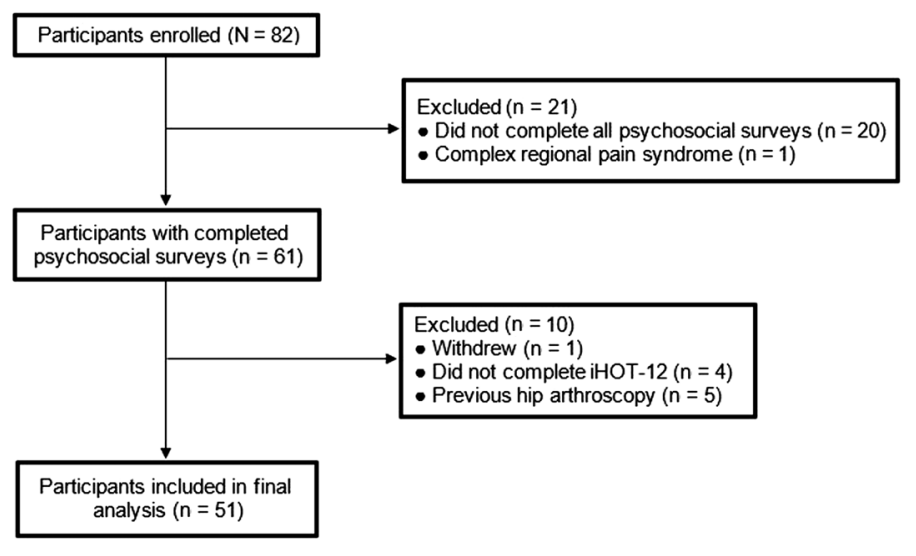

Figure 1 - A flow diagram of participant enrollment and attrition. iHOT-12 indicates 12-item International Hip Outcome Tool.

52, with higher scores demonstrating more frequent or severe catastrophizing. ${ }^{22}$ The PCS has good internal consistency (Cronbach alpha) of .87 and a test-retest reliability of $0.93 .{ }^{22,40}$ The PSEQ asks participants to rate their confidence on a scale from 0 (not at all confident) to 6 (completely confident) in completing tasks despite their current pain. Scores range from 0 to 60 with higher scores representing higher self-efficacy. ${ }^{27}$ The PSEQ has an internal consistency (Cronbach alpha) of .92 and a test-retest reliability of $0.73 .{ }^{27,41,42}$ Finally, fear of movement, or kinesiophobia, was measured with the TSK, which has an internal consistency (Cronbach alpha) of .76 and a test-retest reliability of $0.82 .{ }^{35}$ The TSK is a 17-item scale. Participants read each of the statements and rate them on a scale from 1 (strongly disagree) to 4 (strongly agree). Higher scores indicate greater fear. Self-reported hip function was measured using the iHOT-12. Participants read each of the 12 statements regarding hip-related function and quality of life on a 100-mm VAS. On this scale, 0 represents the worst function and quality of life, and 100 represents the best possible function and quality of life. Lower scores indicate more significant impairment. The iHOT-12 is reliable, valid, and responsive to change with a test-retest reliability of $0.89 .{ }^{43}$

Measures of hip joint morphology (lateral center-edge angle [LCEA] of Wiberg and alpha angle) were included so that the authors could account for the potential relationships between more severe hip joint morphology and psychosocial health, pain, and function. Anteroposterior pelvis and frog leg lateral radiographs were used to measure LCEAs and alpha angles using McKesson computer-assisted radiographic measurement software (Emageon Inc, Birmingham, AL). ${ }^{44}$ Radiographic analysis was performed by 1 author (K.N.J.). Training on radiographic measurements was provided by co-author and board-certified orthopedic surgeon (S.T.D.). Intraobserver variability was established using 15 sets of hip radiographs unrelated to this study. The author (K.N.J.) measured LCEAs and alpha angles on all 15 sets of radiographs at 2 different time points prior to the start of the study. Intraclass correlation coefficients were established with an intraclass correlation coefficients for intraobserver variability from .89 to .91.

\section{Statistical Analysis}

Participant demographics, clinical characteristics, and patientreported outcome scores were reported. Shapiro-Wilk tests were used to assess the normality of the variables of interest. The majority of variables (age, body mass index [BMI], duration of symptoms, VAS-pain during activity, PCS, PSEQ, and LCEA) followed a nonnormal distribution. As such, the relationships between PCS, PSEQ, TSK, VAS-pain, and iHOT-12 were examined using Spearman rank-order correlations. Clinically meaningful correlations were considered as a rho of .5 or greater. ${ }^{45}$ Independent $t$ tests were used to compare VAS-pain at rest, TSK, alpha angle, and iHOT-12 between individuals with and without self-reported depression and/or anxiety (Bonferroni correction; $P \leq .01$ ). Mann-Whitney $U$ tests were used to compare age, BMI, duration of symptoms, VAS-pain during activity, PCS, PSEQ, and LCEA between individuals with and without self-reported depression and/or anxiety (Bonferroni correction; $P \leq .01$ ). Hedge $g$ effect sizes and 95\% confidence intervals were included for comparisons between groups. An effect size of 0.8 was considered large, 0.5 was medium, and 0.2 was small. ${ }^{45}$ All analyses were performed using SPSS Statistics (version 24; IBM Corp, Armonk, NY).

\section{Results}

Approximately $35 \%$ of the 51 participants self-reported depression and/or anxiety $(18 / 51 ; 35.3 \%$; Table 1$)$. No demographics, measures of joint morphology, or patient-reported outcome measures differed between men and women $(P \geq .13)$. Clinically meaningful correlations $(\rho \geq .5)$ were found between the iHOT-12 and pain during activity, PSEQ, and TSK (Table 2). Significant correlations, though just below the cutoff for clinical meaningfulness, were also found between PSEQ and pain at rest and pain during activity. Age, BMI, duration of symptoms, LCEA, and alpha angle were not meaningfully correlated with any of the variables of interest $(\rho \leq$ -.32). Participants with self-reported depression and/or anxiety had significantly higher PCS scores and lower PSEQ scores $(P \leq .01)$. Differences in PCS and PSEQ scores were supported by large effect sizes (Table 3). They did not differ in terms of sex, age, BMI, or joint morphology. Likewise, they did not report worse pain or function.

\section{Table 1 Participant Demographics and Descriptive Information for Variables of Interest}

\begin{tabular}{lc}
\hline Variable & $\mathbf{N}=\mathbf{5 1}$ \\
\hline Age, mean (range) & $35.7(15-56)$ \\
Sex, n (\%) & \\
$\quad$ Male & $9(17.6)$ \\
$\quad$ Female & $42(82.4)$ \\
Self-reported depression and/or anxiety, n (\%) & $18(35.3)$ \\
BMI & $27.1(4.9)$ \\
Duration of symptoms (months), mean (range) & $30.5(1-132)$ \\
Pain at rest & $4.1(2.2)$ \\
Pain during activity & $7.3(2.0)$ \\
PCS & $20.1(13.3)$ \\
TSK & $42.9(6.5)$ \\
PSEQ & $37.8(13.1)$ \\
iHOT-12 & $25.7(16.6)$ \\
LCEA & $30.6^{\circ}\left(7.3^{\circ}\right)$ \\
Alpha angle & $60.8^{\circ}\left(12.2^{\circ}\right)$ \\
\hline
\end{tabular}

Abbreviations: BMI, body mass index; iHOT-12, 12-item International Hip Outcome Tool; LCEA, lateral center-edge angle; PCS, Pain Catastrophizing Scale; PSEQ, Pain Self-Efficacy Questionnaire; TSK, Tampa Scale for Kinesiophobia. Note: Values are presented as mean (SD). 
Table 2 Relationships Between PCS Scores, PSEQ scores, TSK Scores, VAS-Pain (at Rest and During Activity), and iHOT-12 Scores Were Examined Using Spearman Rank-Order Correlations

\begin{tabular}{lccccc}
\hline Variable & VAS-pain (rest) & VAS-pain (activity) & iHOT-12 & TSK & PSEQ \\
\hline PCS & $.30(.04)^{*}$ & $.28(.05)^{*}$ & $-.34(.02)^{*}$ & $\mathbf{. 6 1}(<. \mathbf{0 0 1})^{*}$ & $-.38(.006)^{*}$ \\
PSEQ & $-.43(.002)^{*}$ & $-.46(.001)^{*}$ & $\mathbf{. 7 1}(<. \mathbf{0 0 1})^{*}$ &.$- .56(<.001)^{*}$ & - \\
TSK & $.07(.64)$ & $.16(.28)$ & $-.52(<.001)^{*}$ & - & - \\
iHOT-12 & $-.37(.01)^{*}$ & $-.57(<.001)^{*}$ & - & - & - \\
VAS-pain (activity) & $\mathbf{. 5 3}(<.001)^{*}$ & - & - & - & - \\
\hline
\end{tabular}

Abbreviations: iHOT-12, 12-item International Hip Outcome Tool; PCS, Pain Catastrophizing Scale; PSEQ, Pain Self-Efficacy Questionnaire; TSK, Tampa Scale for Kinesiophobia; VAS, visual analog scale. Note: Bold values signifies a potentially clinically meaningful correlation. Reported as rho $(P$ value).

*Significant correlation at the .05 level.

Table 3 Differences Between Demographics, Psychosocial Factors, Pain, and Function Between Participants With and Without Self-Reported Depression and/or Anxiety (Reported as Mean [SD])

\begin{tabular}{|c|c|c|c|c|c|}
\hline Variable & $\begin{array}{l}\text { Self-reported depression } \\
\text { and/or anxiety }\end{array}$ & $\begin{array}{c}\text { No self-reported depression } \\
\text { and/or anxiety }\end{array}$ & $P$ value & Hedges $\mathbf{g}$ & $95 \% \mathrm{Cl}$ \\
\hline Age & $37.3(11.6)$ & $34.8(11.6)$ & .43 & 0.21 & -0.36 to 0.79 \\
\hline $\operatorname{Sex}(M / F)$ & $2 / 16$ & $7 / 26$ & .46 & - & - \\
\hline BMI & $27.7(3.9)$ & $26.9(5.4)$ & .20 & 0.16 & -0.41 to 0.74 \\
\hline DOS & $34.0(32.3)$ & $28.7(31.4)$ & .26 & 0.16 & -0.41 to 0.74 \\
\hline Pain at rest & $4.5(2.3)$ & $4.0(2.2)$ & .42 & 0.22 & -0.35 to 0.80 \\
\hline Pain during activity & $8.0(1.9)$ & $7.0(2.0)$ & .10 & 0.50 & -0.08 to 1.09 \\
\hline PCS & $27.1(15.4)$ & $16.3(10.4)$ & $.01 *$ & 0.86 & 0.27 to 1.47 \\
\hline TSK & $45.6(6.9)$ & $41.4(5.9)$ & $.04 *$ & 0.66 & 0.08 to 1.26 \\
\hline PSEQ & $31.1(12.1)$ & $41.6(12.3)$ & $.006^{*}$ & -0.85 & -1.45 to -0.25 \\
\hline LCEA & $28.9(7.5)$ & $31.5(7.1)$ & .39 & -0.35 & -0.94 to 0.22 \\
\hline Alpha angle & $60.9(12.1)$ & $60.7(12.4)$ & .95 & 0.02 & -0.56 to 0.59 \\
\hline iHOT-12 & $20.7(16.7)$ & $28.4(16.1)$ & .12 & -0.46 & -1.05 to 0.11 \\
\hline
\end{tabular}

Abbreviations: BMI, body mass index; CI, confidence interval; DOS, duration of symptoms; F, female; iHOT-12, 12-item International Hip Outcome Tool; LCEA, lateral center-edge angle; M, male; PCS, Pain Catastrophizing Scale; PSEQ, Pain Self-Efficacy Questionnaire; TSK, Tampa Scale for Kinesiophobia. $*$ Statistically significant $\leq .01$.

\section{Discussion}

This study aimed to examine the relationships between pain catastrophizing, self-efficacy, kinesiophobia, pain, and function in individuals with FAIS. Our hypothesis that higher pain catastrophizing, lower self-efficacy, and higher kinesiophobia would be related with worse pain, and function was partially supported. Lower self-efficacy was related with worse pain and function, and higher kinesiophobia was related with worse function. However, higher pain catastrophizing was not meaningfully related with worse pain or function. In addition, this study identified that participants with self-reported depression and/or anxiety had higher pain catastrophizing and lower self-efficacy than those without self-reported depression and/or anxiety.

Pain is complex and can rarely be explained entirely by a single variable; however, the current study demonstrates the importance of considering self-efficacy when evaluating pain in patients with FAIS. Low self-efficacy is related to a variety of poor outcomes in both nonoperative management and postoperative rehabilitation of musculoskeletal conditions. ${ }^{36,46-48}$ These consequences range from increased pain and disability to reduced function. Though findings from this study cannot be extrapolated to posthip arthroscopy outcomes, the characterizations of poor psychosocial health provides guidance regarding potential treatment targets in the future. Evidence-based interventions such as goal setting have shown success in improving self-efficacy. ${ }^{49-51}$ Beneficially, goal setting is an intervention accessible to any allied health professionals (ie, athletic trainers, physical therapists, nurses, etc), which extends its applicability in the rehabilitative setting.

In the current study, both low self-efficacy and high kinesiophobia were related with worse function in individuals with FAIS ( $\rho=.71$ and -.52 , respectively). However, the authors did not find a clinically meaningful correlation between pain catastrophizing and function $(\rho=-.34)$. The mean PCS score in the current study (20.1 [13.3]) falls between the 2 previously reported in individuals with FAIS (23.7 [11.8] and 17.81 [10.13]). ${ }^{11,33}$ Given that Clapp et $\mathrm{al}^{33}$ found an association between higher baseline PCS scores and not achieving a minimally clinically important difference for a hipspecific outcome score 1 year following hip arthroscopy, the implications of high pain catastrophizing may be found in longitudinal assessments of pain or functional outcomes.

Findings from this study support that patients' perception and their injury influences their pain experience and functional status. This is consistent with Woby's fear-avoidance model, which suggests these psychosocial factors play an integral role in 
avoidance or confrontation of injury recovery. ${ }^{36}$ As such, the current findings have important implications for treating physicians and rehabilitation specialists. Clinicians should be aware of these associations and should consider screening for low selfefficacy or high kinesiophobia in individuals not responding to normal therapeutic interventions. Future studies should examine adjunct allied health interventions aimed at increasing self-efficacy and lowering kinesiophobia. Such treatment interventions (eg, pain neuroscience education, mindfulness, and goal setting) have demonstrated success in increasing self-efficacy and lowering kinesiophobia in other orthopedic populations. ${ }^{49-54}$ The current findings add support to a growing body of literature warranting psychosocial intervention studies in patients with FAIS.

\section{Psychosocial and Mental Health in FAIS}

A growing body of literature in patients with FAIS suggests those with self-reported mental health disorders, specifically anxiety or depression, present with worse pain, have worse postoperative pain and function, and an increased prevalence of prolonged opioid use. ${ }^{13-18,37}$ Findings from the current study suggest individuals with self-reported depression and/or anxiety have higher pain catastrophizing and lower self-efficacy. Interestingly they did not report worse pain or function. The authors hypothesize that poor psychosocial health may be the intermediary between mental health disorders and pain and functional outcomes. To support this hypothesis, a 2015 study by Skidmore et $\mathrm{al}^{46}$ identified that in patients with chronic low back pain, a change in depressive symptoms predicted changes in pain, and this relationship was mediated by pain selfefficacy. Those patients that were able to increase their confidence to perform activities, despite their current pain, displayed fewer depressive symptoms and reported less pain following a 4-week rehabilitation program. Future work should focus on understanding the complex interactions between mental health disorders, psychosocial factors, and pain and functional outcomes in individuals with FAIS.

\section{Limitations}

This study was not without limitations. First, as with any crosssectional study design, the authors cannot assume cause and effect of poor psychosocial health on pain and function. The lack of a comparison arm is a limitation of these findings, and as such, future studies should include both a group of patients with FAIS who respond favorably to conservative treatment and a control group of healthy age-matched patients. A more thorough comparison could include an asymptomatic group of matched patients with similar hip morphology. The authors are currently performing a longitudinal study to assess the effect of pain catastrophizing, self-efficacy, and kinesiophobia on posthip arthroscopy outcomes; however, at this time, the lack of correlation to long-term outcomes is a notable limitation. Though no demographics, measures of joint morphology, or patient-reported outcome measures (including psychosocial, pain, and functional outcomes) differed between the sexes $(P \geq .13)$, the predominantly female cohort is a limitation to the external validity of these results. Depression and/or anxiety were selfreported. Thus, participants in this group may not have met the clinical diagnostic criteria for having depression and/or anxiety. Finally, the heterogeneity of this patient population must be noted. The authors attempted to control for this by excluding patients who did not complete the standard of care nonoperative treatment and those who were undergoing revision arthroscopy.

\section{Conclusion}

Low self-efficacy and high kinesiophobia were related with worse function in individuals with FAIS. Furthermore, low-self efficacy and low function were related with worse pain during activity, and low self-efficacy was related with worse pain at rest. About 35.3\% of participants self-reported depression and/or anxiety, and those individuals had lower self-efficacy and higher pain catastrophizing. As mental health disorders, low self-efficacy, and high kinesiophobia have been linked to poorer outcomes in other orthopedic populations, future studies should focus on evaluating the effect of self-efficacy and kinesiophobia on longitudinal treatment outcomes as well as on the development of treatment strategies and interventions aimed at improving the likelihood of a successful clinical outcome for these high-risk groups.

\section{Acknowledgments}

S.T.D. reports grants and personal fees from Smith and Nephew, grants and personal fees from Zimmer/Biomet, grants from Stryker, and grants from BONESUPPORT. C.A.J. reports grants from Medtronic, grants from Smith and Nephew, grants and personal fees from Flexion Therapeutics.

\section{References}

1. Griffin DR, Dickenson EJ, O'Donnell J, et al. The Warwick agreement on femoroacetabular impingement syndrome (FAI syndrome): an international consensus statement. Br J Sports Med. 2016;50(19):11691176. PubMed ID: 27629403 doi:10.1136/bjsports-2016-096743

2. Lahner M, Walter PA, von Schulze Pellengahr C, Hagen M, von Engelhardt LV, Lukas C. Comparative study of the femoroacetabular impingement (FAI) prevalence in male semiprofessional and amateur soccer players. Arch Orthop Trauma Surg. 2014;134(8):1135-1141. PubMed ID: 24858466 doi:10.1007/s00402-014-2008-6

3. Agricola R, Heijboer MP, Ginai AZ, et al. A cam deformity is gradually acquired during skeletal maturation in adolescent and young male soccer players: a prospective study with minimum 2year follow-up. Am J Sports Med. 2014;42(4):798-806. PubMed ID: 24585362 doi: $10.1177 / 0363546514524364$

4. Kivlan BR, Nho SJ, Christoforetti JJ, et al. Multicenter outcomes after hip arthroscopy: epidemiology (MASH study group). What are we seeing in the office, and who are we choosing to treat? Am J Orthop. 2017;46(1):35-41. PubMed ID: 28235111

5. Clohisy JC, Baca G, Beaulé PE, et al. Descriptive epidemiology of femoroacetabular impingement: a North American cohort of patients undergoing surgery. Am J Sports Med. 2013;41(6):1348-1356. PubMed ID: 23669751 doi:10.1177/0363546513488861

6. Kahlenberg CA, Han B, Patel RM, Deshmane PP, Terry MA. Time and cost of diagnosis for symptomatic femoroacetabular impingement. Orthop J Sports Med. 2014;2(3):2325967114523916. PubMed ID: 26535305 doi:10.1177/2325967114523916

7. Levy DM, Kuhns BD, Chahal J, Philippon MJ, Kelly BT, Nho SJ. Hip arthroscopy outcomes with respect to patient acceptable symptomatic state and minimal clinically important difference. Arthroscopy. 2016;32(9):1877-1886. PubMed ID: 27324968 doi:10.1016/j. arthro.2016.05.014

8. King MG, Lawrenson PR, Semciw AI, Middleton KJ, Crossley KM. Lower limb biomechanics in femoroacetabular impingement syndrome: a systematic review and meta-analysis. Br J Sports Med. 2018;52(9):566-580. PubMed ID: 29439949 doi:10.1136/bjsports2017-097839 
9. Jones DM, Crossley KM, Ackerman IN, et al. Physical activity following hip arthroscopy in young and middle-aged adults: a systematic review. Sports Med. 2020;6(1):1-15. PubMed ID: 31993831 doi:10.1186/s40798-020-0234-8

10. Mallets E, Turner A, Durbin J, Bader A, Murray L. Short-term outcomes of conservative treatment for femoroacetabular impingement: a systematic review and meta-analysis. Int J Sports Phys Ther. 2019;14(4):514-524. PubMed ID: 31440404

11. Mansell NS, Rhon DI, Meyer J, Slevin JM, Marchant BG. Arthroscopic surgery or physical therapy for patients with femoroacetabular impingement syndrome: a randomized controlled trial with 2-year follow-up. Am J Sports Med. 2018;46(6):1306-1314. PubMed ID: 29443538 doi:10.1177/0363546517751912

12. Hunt D, Prather H, Hayes MH, Clohisy JC. Clinical outcomes analysis of conservative and surgical treatment of patients with clinical indications of prearthritic, intra-articular hip disorders. $P M \& R$. 2012;4(7):479487. PubMed ID: 22595328 doi:10.1016/j.pmrj.2012.03.012

13. Stone AV, Malloy P, Beck EC, et al. Predictors of persistent postoperative pain at minimum 2 years after arthroscopic treatment of femoroacetabular impingement. Am J Sports Med. 2019;47(3):552-559. PubMed ID: 30822125 doi:10.1177/0363546518817538

14. Stone AV, Beck EC, Malloy P, et al. Preoperative predictors of achieving clinically significant athletic functional status after hip arthroscopy for femoroacetabular impingement at minimum 2-year follow-up. Arthroscopy. 2019;35(11):3049-3056. PubMed ID: 31395395 doi:10.1016/j.arthro.2019.05.022

15. Jochimsen KN, Magnuson JA, Kocan KR, et al. Anxiety and depression are associated with lower preoperative quality of life and function but not duration of symptoms in patients with femoroacetabular impingement syndrome. J Hip Preserv Surg. 2019;6(3): 207-213.

16. Jacobs CA, Burnham JM, Jochimsen KN, Molina D, Hamilton DA, Duncan ST. Preoperative symptoms in femoroacetabular impingement patients are more related to mental health scores than the severity of labral tear or magnitude of bony deformity. J Arthroplasty. 2017;32(12): 3603-3606. PubMed ID: 28739309 doi:10.1016/j.arth.2017.06.053

17. Westermann RW, Lynch TS, Jones MH, et al. Predictors of hip pain and function in femoroacetabular impingement: a prospective cohort analysis. Orthop J Sports Med. 2017;5(9):2325967117726 521. PubMed ID: 28944250 doi:10.1177/2325967117726521

18. Cheng AL, Schwabe M, Doering MM, Colditz GA, Prather H. The effect of psychological impairment on outcomes in patients with prearthritic hip disorders: a systematic review and meta-analysis. Am J Sports Med. 2020;48(10):2563-2571. PubMed ID: 31829034 doi: $10.1177 / 0363546519883246$

19. Wylie JD, Suter T, Potter MQ, Granger EK, Tashjian RZ. Mental health has a stronger association with patient-reported shoulder pain and function than tear size in patients with full-thickness rotator cuff tears. J Bone Joint Surg Am. 2016;98(4):251-256. PubMed ID: 26888672 doi:10.2106/JBJS.O.00444

20. Kim KW, Han JW, Cho HJ, et al. Association between comorbid depression and osteoarthritis symptom severity in patients with knee osteoarthritis. J Bone Joint Surg Am. 2011;93(6):556-563. PubMed ID: 21411706 doi:10.2106/JBJS.I.01344

21. Jacobs CA, Christensen CP, Karthikeyan T. Chronic non-orthopedic conditions more common in patients with less severe degenerative changes that have elected to undergo total knee arthroplasty. J Arthroplasty. 2015;30(7):1146-1149. PubMed ID: 25702593 doi:10.1016/j.arth.2015.01.051

22. Sullivan MJ, Bishop SR, Pivik J. The pain catastrophizing scale: development and validation. Psychol Assess. 1995;7(4):524.
23. Guite JW, McCue RL, Sherker JL, Sherry DD, Rose JB. Relationships among pain, protective parental responses, and disability for adolescents with chronic musculoskeletal pain: the mediating role of pain catastrophizing. Clin J Pain. 2011;27(9):775-781. PubMed ID: 21593664 doi:10.1097/AJP.0b013e31821d8fb4

24. Talaei-Khoei M, Fischerauer SF, Lee SG, Ring D, Vranceanu AM. Pain catastrophizing mediates the effect of psychological inflexibility on pain intensity and upper extremity physical function in patients with upper extremity illness. Pain Pract. 2017;17(1):129-140. PubMed ID: 27739246 doi:10.1111/papr.12494

25. Vranceanu AM, Bachoura A, Weening A, Vrahas M, Smith RM, Ring DJJ. Psychological factors predict disability and pain intensity after skeletal trauma. Bone Joint J. 2014;96(3):e20. PubMed ID: 24500592 doi:10.2106/JBJS.L.00479

26. Lewis GN, Rice DA, McNair PJ, Kluger M. Predictors of persistent pain after total knee arthroplasty: a systematic review and metaanalysis. Br J Anaesth. 2015;114(4):551-561. PubMed ID: 25542191 doi:10.1093/bja/aeu441

27. Nicholas MK. The pain self-efficacy questionnaire: taking pain into account. Eur J Pain. 2007;11(2):153-163. PubMed ID: 16446108 doi:10.1016/j.ejpain.2005.12.008

28. van den Akker-Scheek I, Stevens M, Groothoff JW, Bulstra SK, Zijlstra W. Preoperative or postoperative self-efficacy: which is a better predictor of outcome after total hip or knee arthroplasty? Patient Educ Couns. 2007;66(1):92-99.

29. Costal LdCM, Maherl CG, McAuleyl JH, Hancockl MJ, Smeetsl RJ. Self-efficacy is more important than fear of movement in mediating the relationship between pain and disability in chronic low back pain. Eur J Pain. 2011;15(2):213-219. PubMed ID: 20655254 doi:10. 1016/j.ejpain.2010.06.014

30. Kvist J, Ek A, Sporrstedt K, Good L. Fear of re-injury: a hindrance for returning to sports after anterior cruciate ligament reconstruction. Knee Surg Sports Traumatol Arthrosc. 2005;13(5):393-397. PubMed ID: 15703963 doi:10.1007/s00167-004-0591-8

31. Glaws KR, Ellis TJ, Hewett TE, Di Stasi SL. Return to sport rates in physically active individuals six months after arthroscopy for femoroacetabular impingement syndrome. J Sport Rehabil. 2019; 28(6):570-575. PubMed ID: 29651906 doi:10.1123/jsr.20170138

32. Riff AJ, Ukwuani G, Clapp I, Movassaghi K, Kelly DM, Nho SJ. High rate of return to high-intensity interval training after arthroscopic management of femoroacetabular impingement syndrome. Am J Sports Med. 2018;46(11):2594-2600. PubMed ID: 29869890 doi:10.1177/0363546518776638

33. Clapp I, Nwachukwu BU, Beck EC, et al. What is the role of kinesiophobia and pain catastrophizing in outcomes after hip arthroscopy for femoroacetabular impingement syndrome? Arthrosc Sport Med Rehabil. 2020;2(2):e97-e104. PubMed ID: 32368745 doi:10. 1016/j.asmr.2019.12.001

34. Hwang CT, Van Dillen LR, Haroutounian S. Do changes in sensory processing precede low back pain development in healthy individuals? Clin J Pain. 2018;34(6):525-531. PubMed ID: 29016389 doi:10.1097/AJP.0000000000000563

35. Woby SR, Roach NK, Urmston M, Watson PJ. Psychometric properties of the TSK-11: a shortened version of the Tampa Scale for Kinesiophobia. Pain. 2005;117(1-2):137-144. PubMed ID: 16055269 doi:10.1016/j.pain.2005.05.029

36. Woby SR, Urmston M, Watson PJ. Self-efficacy mediates the relation between pain-related fear and outcome in chronic low back pain patients. Eur J Pain. 2007;11(7):711-718. PubMed ID: 17218132 doi:10.1016/j.ejpain.2006.10.009 
37. Jacobs CA, Hawk GS, Jochimsen KN, et al. Depression and anxiety are associated with increased health care costs and opioid use for patients with femoroacetabular impingement undergoing hip arthroscopy: analysis of a claims database. Arthroscopy. 2020;36(3): 745-750. PubMed ID: 31924382 doi:10.1016/j.arthro.2019.09.048

38. Sullivan MJ, D'Eon JL. Relation between catastrophizing and depression in chronic pain patients. J Abnorm Psychol. 1990;99(3):260. PubMed ID: 2145334 doi:10.1037//0021-843x.99.3.260

39. Wylde V, Dixon S, Blom AW. The role of preoperative self-efficacy in predicting outcome after total knee replacement. Musculoskeletal Care. 2012;10(2):110-118. PubMed ID: 22368121 doi:10.1002/msc.1008

40. Osman A, Barrios FX, Gutierrez PM, Kopper BA, Merrifield T, Grittmann L. The pain catastrophizing scale: further psychometric evaluation with adult samples. J Behav Med. 2000;23(4):351-365. PubMed ID: 10984864 doi:10.1023/a:1005548801037

41. Vong SK, Cheing GL, Chan CC, Chan F, Leung AS. Measurement structure of the pain self-efficacy questionnaire in a sample of Chinese patients with chronic pain. Clin Rehabil. 2009;23(11):1034-1043. PubMed ID: 19656814 doi:10.1177/0269215509337448

42. Nicholas MK, Asghari A, Blyth FM. What do the numbers mean? Normative data in chronic pain measures. Pain. 2008;134(1-2):158173. PubMed ID: 17532138 doi:10.1016/j.pain.2007.04.007

43. Griffin DR, Parsons N, Mohtadi NGH, Safran MR. Network MA of the HOR. A short version of the international hip outcome tool (iHOT-12) for use in routine clinical practice. Arthroscopy. 2012;28(5):611-618. PubMed ID: 22542434 doi:10.1016/j.arthro.2012.02.027

44. Duncan CP, Carlisle J, Clohisy JC. Radiographic evaluation of the hip. In: Clohisy JC, Beaule PE, Della Valle CJ, eds. The Adult Hip: Hip Preservation Surgery. Philadelphia, PA: Wolters Kluwer; 2014:131-143.

45. Cohen J. Statistical Power Analysis for the Behavioral Sciences. New York, NY: Academic Press; 2013.

46. Skidmore JR, Koenig AL, Dyson SJ, Kupper AE, Garner MJ, Keller CJ. Pain self-efficacy mediates the relationship between depressive symptoms and pain severity. Clin J Pain. 2015;31(2):137-144. PubMed ID: 24751545 doi:10.1097/AJP.0000000000000094
47. Thomeé P, Währborg P, Börjesson M, Thomeé R, Eriksson BI, Karlsson J. Self-efficacy of knee function as a pre-operative predictor of outcome 1 year after anterior cruciate ligament reconstruction. Knee Surg Sport Traumatol Arthrosc. 2008;16(2):118-127. PubMed ID: 18034333 doi:10.1007/s00167-007-0433-6

48. Everhart JS, Best TM, Flanigan DC. Psychological predictors of anterior cruciate ligament reconstruction outcomes: a systematic review. Knee Surg Sport Traumatol Arthrosc. 2015;23(3):752-762. PubMed ID: 24126701 doi:10.1007/s00167-013-2699-1

49. Coppack RJ, Kristensen J, Karageorghis CI. Use of a goal setting intervention to increase adherence to low back pain rehabilitation: a randomized controlled trial. Clin Rehabil. 2012;26(11):1032-1042. PubMed ID: 22357799 doi:10.1177/0269215512436613

50. Evans L, Hardy L. Injury rehabilitation: a goal-setting intervention study. Res Q Exercise Sport. 2002;73(3):310-319. PubMed ID: 12230338 doi:10.1080/02701367.2002.10609025

51. Lucas A, Borce KN, Fast AW, Walker-Tweed J. Effect of goal setting using a visual display on patient quality of life and self-efficacy. $J$ Nurs Care Qual. 2018;33(2):100-103. PubMed ID: 28915222 doi:10. 1097/NCQ.0000000000000293

52. Jay K, Brandt M, Jakobsen MD, et al. Ten weeks of physicalcognitive-mindfulness training reduces fear-avoidance beliefs about work-related activity: randomized controlled trial. Medicine. 2016; 95(34):e3945-e3945. PubMed ID: 27559939 doi:10.1097/MD. 0000000000003945

53. Bodes Pardo G, Lluch Girbes E, Roussel NA, Gallego Izquierdo T, Jimenez Penick V, Pecos Martin D. Pain neurophysiology education and therapeutic exercise for patients with chronic low back pain: a single-blind randomized controlled trial. Arch Phys Med Rehabil. 2018;99(2):338-347. PubMed ID: 29138049 doi:10.1016/j.apmr. 2017.10.016

54. Louw A, Zimney K, Puentedura EJ, Diener I. The efficacy of pain neuroscience education on musculoskeletal pain: a systematic review of the literature. Physiother Theory Pract. 2016;32(5):332-355. PubMed ID: 27351541 doi:10.1080/09593985.2016.1194646 